\title{
A Scalable and License Free 5G Internet of Radio Light Architecture for Services in Homes \& Businesses
}

\author{
John Cosmas ${ }^{1}$, Ben Meunier ${ }^{1}$, Kareem Ali $^{1}$, Nawar Jawad ${ }^{1}$, Mukhald Salih ${ }^{1}$, Hong-Ying Meng ${ }^{1}$, Martin Ganley ${ }^{2}$, \\ James Gbadamosi ${ }^{2}$, Atanas Savov ${ }^{2}$, Zion Hadad ${ }^{3}$, Baruch Globen $^{3}$, Haluk Gokmen ${ }^{4}$, Sibel Malkos ${ }^{4}$, Memduh Emre \\ Cakan $^{4}$, Harilaos Koumaras ${ }^{5}$, Michael-Alexandros Kourtis ${ }^{5}$, Christos Sakkas ${ }^{5}$, Eliron Salomon ${ }^{6}$, Yoav Avinoam ${ }^{6}$, \\ Daniel Negru', Mathias Lacaud ${ }^{7}$, Yue Zhang ${ }^{8}$, Li-Ke Huang ${ }^{9}$, Rudolf Zetik ${ }^{10}$, Krzysztof Cabaj ${ }^{11}$, Wojciech \\ Mazurczyk $^{11}$, Xun Zhang ${ }^{12}$, Moshe Ran ${ }^{13}$, Dror Malka ${ }^{13}$, Adam Kapovits ${ }^{14}$
}

\begin{abstract}
Brunel University ${ }^{1}$, Building Research Establishment ${ }^{2}$, Runel plc $^{3}$, Arcelik A. $S^{4}$, National Centre for Scientific Research Demokritos ${ }^{5}$, Holon Institute of Technology ${ }^{6}$, Joada $^{7}$, University of Bedfordshire ${ }^{8}$, Cobham Wireless ${ }^{9}$, FHGIIS $^{10}$, Warsaw University of Technology ${ }^{11}$, ISEP $^{12}$, MostlyTek $^{13}$, Eurescom $^{14}$
\end{abstract}

Email: john.cosmas@brunel.ac.uk

\begin{abstract}
In this paper we present a 5G Internet Radio-Light (IoRL) architecture for homes that can be readily deployed because it utilizes unlicensed visible light and millimeter wave part of the spectrum, which does not require Mobile Network Operator (MNO) permission to deploy and which is used to provide inhabitants of houses with accurate location, interaction, access to Internet and Cloud based services such as high resolution video on a Tablet $\mathrm{PC}$. The paper describes the home use cases and the IoRL architecture.
\end{abstract}

Keywords-Homes, Visible Light Communications, mm Wave Communications, Network Function Virtualisation, Software Defined Networks, 5G System Architecture.

\section{INTRODUCTION}

One of the growing problems in buildings is interference due to congestion in WLANs. This has been due to an ever increasing capacity demand for data traffic from Smartphones, mobile PCs and Tablets with voice traffic occupying only a fraction of the total capacity demand [1]. This interference has been compounded by the range of different wireless systems in homes and businesses, namely: electronic equipment such as microwave ovens, cordless phones, wireless headsets, Zigbee, ZWave, Bluetooth devices, surveillance cameras and other wireless radio networks. Thus the main motivation for homes and businesses has been to switch to home networks that use regulated spectrum such as HeNBs to avoid interference problems.

The deployment of HeNB small cells requires the permission of MNOs, due to their potential to interfere with the main eNB transmitted signal, but the MNOs only have the resources to approve installation for their larger business clients (with 100 employees and above) and neglect their smaller clients, who are by far the largest group in the market place [2]. These smaller clients then become frustrated and return to using WiFi! This trend is being experienced worldwide.
Furthermore larger clients, who wish to use HeNB small cells in their premises, require separate infrastructure for every MNO that is providing coverage in a building, which is very costly and inconvenient to the client. This has led to the concept of the sharing of mobile network resources, such as with Multi Operator Radio Access Network (MORAN) HeNB systems, and the concept of improving the coverage within buildings such as with Distributed Antenna Systems (DAS), both of which continue to be costly options for the client.

Clearly with a compound annual growth rate (CAGR) of $57 \%$ with forecasts of 24.3 Exabytes mobile data traffic by 2019 [3], the competition for spectrum, which is already congested, will only intensify. However $80 \%$ of this traffic is generated indoors and is growing $20 \%$ faster each year than outdoor wireless traffic [4] [5]. Since fewer than $2 \%$ of commercial and public buildings are currently covered by dedicated wireless indoor solutions [6], the introduction of a commercial network solution in the unlicensed millimeter wave $(30 \mathrm{GHz}-300 \mathrm{GHz})$ and Visible Light $(400 \mathrm{~T} \mathrm{~Hz}-800$ $\mathrm{THz}$ ) spectrum for mobile networks could have an enormous impact on the indoor building market.

\section{TECHNOLOGY BACKGROUND}

MmWave signals exhibit reduced diffraction and a more specular propagation than their microwave counterparts, and hence they are much more susceptible to blockages. In that sense mm-wave line-of-sight propagation might be likened to that of visible light. This results in a nearly bimodal channel depending on the presence or absence of line-of-sight. A main difference between microwave and mmWave frequencies is the sensitivity to blockages: the results in [7], for instance, indicate a path loss exponent of 2 for line-of-sight propagation but 4 (plus additional power loss) for non-line-of-sight. As a result of the sensitivity to blockages, a given link can rapidly transition from usable to unusable and, unlike small-scale fading, large-scale obstructions cannot be circumvented with standard small-scale diversity countermeasures. 
Mm Wave wireless signals propagation through free space, experience attenuation so usable path lengths are short, roughly 100 to 200 meters. Such distances could be accommodated with the smaller cell sizes envisioned in $5 \mathrm{G}$, but there are numerous other impediments. Buildings and the objects in and around them, including people, block the signal. Rain and foliage further attenuate millimeter waves, and diffraction-which can bend longer wavelengths around occluding objects-is far less effective. Even surfaces that might be conveniently nicely reflective at longer wavelengths appear rougher to millimeter waves, and so diffuse the signal. Since signals cannot readily propagate through outdoor building materials, then indoor networks will be isolated from outdoor networks and this suggests that access points may need to be installed for handovers at entrances of commercial and residential buildings [7].

WiGig Wireless LAN IEEE 802.11ad technology is a backwards-compatible extension to the IEEE 802.11-2012 specification that adds a new MAC/PHY to provide short range, high capacity links in the $60 \mathrm{GHz}$ unlicensed band. It could be considered as an interesting technical solution for Wireless Home networking protocol as it has been rapidly evolving to support the increasing demand for high data rates, with the standard providing $6.7 \mathrm{~Gb} / \mathrm{s}$ using $\mathrm{GHz}$ of bandwidth at $60 \mathrm{GHz}$ mmWave frequencies [8]. In current Wi-Fi systems, interworking between WiGig and LTE/LTE-A systems is not supported, although it is badly needed due to users' frequent mobility between the coverage areas of Wi-Fi access points and mobile networks. Therefore the solution, as proposed by [9], could be used to manage handovers between mobile network and the WiGig Home Network and between the different rooms within the WiGig Home Network.

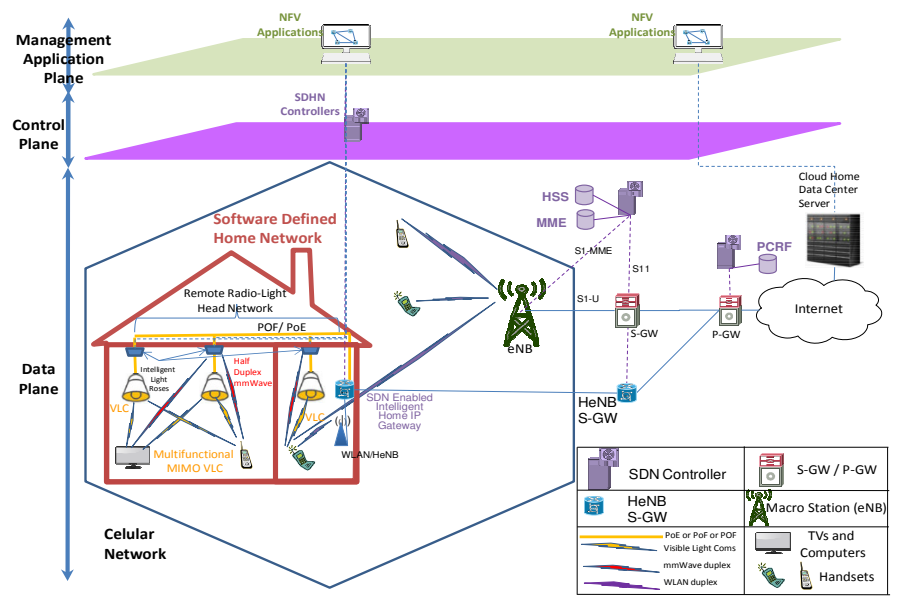

Figure 1: IoRL Network Architecture

Internet of Radio-Light (IoRL) architecture is a 5G RadioLight, multi-component carrier, Frequency Division Duplex (FDD) broadband system for buildings, shown in Figure 1, consisting of a VLC downlink channel in the unlicensed $\mathrm{THz}$ spectrum and mm Wave up/downlink channels in unlicensed 30-300 GHz spectrum, which allows wireless communication networks that can provide bitrates greater than $10 \mathrm{Gbits} / \mathrm{sec}$ to be deployed in buildings, latencies of less than $1 \mathrm{~ms}$, location accuracy of less than $10 \mathrm{~cm}$, whilst reducing EMF levels and interference, lowering energy consumption at transmitter/receiver and increasing User Equipment (UE) energy battery lifetime. The project's Software Defined Home Network (SDHN) Architecture, shown in Figure 1, not only allows network service providers to develop Security Monitoring, Energy Saving, Location Sensing, Network Slicing, Lights Configuration, Video and Network Transport Configuration and Network Security applications but also provides the means to locate network operations and management functions between the Intelligent Home IP Gateway (HIPG) and the Cloud Home Data Centre (CHDC) server in a configurable way to meet the different OPEX and CAPEX needs of different Mobile Network Operators (MNOs).

Furthermore it does not require MNO approval for deployment [10]. This step change in performance and flexibility is a very attractive solution for retailers since it will increase their ability to promote their brand and products thereby improving their profitability, which will incentivize them to raise capital to finance the upgrade of their building network infrastructure.

\section{USE CASES}

This section presents home use cases.

\section{A. Entry to Empty and Secured Home}

An approved family member is watching Youtube video on a smartphone and enters the home through the front door. A motion sensor detects his movement and identifies him through the mmWave uplink channel that he is entering the home, authenticates him, switches on the LED lights entrance hall and stair well and automatically hands over the Youtube video app to the VLC home network from the outside network.

\section{B. Multiple Contents Display and Recording}

By VLC technology, multiple contents from different service providers can be downloaded and displayed by different users at home very rapidly. Record ability for the multi channels by multi end-users at home will be supported too e.g. Olympic Games. During the season of the Olympic Games the different programs of sports are displayed during the same time-slot. The end-users at home can watch or record the different sport programs at the same time. By VLC system integrated to TV, end-users will be able to download and display the multiple contents much faster than wired/wireless networking system.

\section{Broadband Office in the Home}

Many home-based or remote working jobs, such as online tutoring, internet research, blogging, freelance writing, transcription services, website design, fundraising management and consultancy, require a computer and Internet use. In this use case a member of the family is working from home and the office computer and Smart Phone is connected to the Internet via IoRL.

\section{Virtual Reality Tourism from your Living Room}

A family member at home is in living room viewing on his/her VR glasses 360 degree 1920×1080 live stream video at 
30fps from traveling family member's Rocoh Theta S camera fixed to a selfie stick in a Museum in Paris. The family member at home is traveling family member's virtual companion who is able to view the 360 degree view of the tourist site.

\section{E. Follow me video watching experience in transit between rooms}

End-user starts to watch a film or content in the living room or any room in the house and then he/she can go to the other room in the house. In that case, he/she wants to continue watching the same video in the other room from another device such as another television or tablet or their own smart phone. The same video or content will be able to be watched by enduser moving from a room to other room automatically (e.g. Mother can start to watch the video on the living room) by following the location of the end user's Smart Phone.

\section{F. Secure Home using Outside Monitoring}

Dedicated in-house cameras or the cameras placed around the house will be send data to VLC integrated television system. The video will be displayed on television set with small screen by Picture in/and Picture (PIP/PAP) to the enduser. End-user can be able to watch the live video coming from cameras on television set. If any emergency situation such as robbery, fire or unexpected weather conditions etc. the alert message will be transmitted to the VLC integrated television system. End-users will be awaked by displaying alert message on the television set and by making the sound from the speakers of the television set.

\section{G. Broadband Media Handover in the Home}

A family member is in the kitchen watching Internet HDTV on her tablet PC using iPlayer using Multiple Source Streaming and also accessing on the Internet co-information on smart phone using Google Chrome.

She moves from the kitchen to the roof conservatory dining room and the Internet TV on her tablet PC automatically hands over from the ground floor kitchen VLC network to the first floor roof conservatory dining room VLC network. She then moves downstairs to the outside into the basement conservatory and the PC automatically hands over from the Home Network to the outside Mobile Network.

\section{H. Eves Dropping and Rogue VLC Transmitter Security Threats}

For homes and small business there are two potential cyber security threats, namely: eavesdropping of the residents' transmitted sensitive data and attempts to forge or repeat some parts of the communication. In the first case an attacker can intercept some arbitrary data, due to nature of the utilized transmission medium. This can be done, for example, from garden through the window or using any physical access to the light signal through the key hole or the crack under the door. The second case, can be potentially very dangerous, as is allows the attacker to physically access home. Moreover, the attacker can introduce rogue VLC transmitter that can be used for attacking residents from outside the house. It must be emphasized that the smart home scenario is relatively more secure than when the same technology is implemented e.g. in public places, for example supermarket or train station, however the threats mentioned above are still feasible.

The initial mitigation techniques to alleviate such threats include: usage of encrypted communication and applying appropriate authentication mechanisms, which prevents replay attacks, even if the attacker is able to eavesdrop some parts of previously conducted transmissions. Appropriate positioning of VLC transmitters can reduce these threats as well. Moreover, detection and monitoring of cyber security incidents can be realized using SDN-based solution which can be crafted with the IoRL architecture and its characteristic features.

\section{SYSTEM ARCHITECTURE}

The IoRL architecture is a layered architecture consisting of four layers namely: Service, Network Function Virtualisation (NFV), Software Defined Network (SDN) and Access. It is an architecture that is more akin to a Radio-Light Home eNodeB suitable for a single building network rather than an EPC suitable for a whole country.

The Service layer is required to run server side applications to stream audio-video, receive, store results on databases and monitor security etc. from a multi-core Cloud Home Data Centre Server (CHDCS) and is required to run mobile apps from User Equipment (UE) i.e. Smart Phones, Tablet PCs, Virtual Reality Headsets and HDTVs.

At the SDN Layer resides the SDN Forwarding Device (FD) to route IP packets to/from their 5G Layer 2/3 Protocol Processors and the Internet or 5G Network Interfaces connected to the SDN Controller. The Network Function Virtualisation Orchestrator (NFVO) invokes various virtual network functions (VNFs) required for an Intelligent Home IP Gateway (IHIPG) such as local access \& mobility management, deep packet inspection, transcoding processes for video streaming and network security functions (e.g. firewall etc.).

The Access Layer consists of six RRLH Controllers. Each RRLH Controller drives up to eight VLC and mmWave RRLH pairs with the same Transmission Block Sub-Frame, thereby providing a Multiple Input Single Output (MISO) transmission on downlink paths and Single Input Multiple Output (MISO) on uplink paths for its coverage area, which is typically a room or floor area of a building.

A UE can obtain direct access to the Internet, by using $5 \mathrm{G}$ protocols on the Access Layer interface to the UE, to deliver IP packets to the Network Layer and thence to the Server Applications in the Service Layer via the Internet. Alternatively the UE obtains access to the Mobile Network Operator's (MNO) Evolved Packet Core (EPC), by using 5G protocols on the Access Layer interfaces to both the UE and EPC, to deliver IP packets to the Network Layer and thence to the applications supported by the MNO. This latter approach allows applications, such as Facebook, on a Smart phone to be accessed on both the outside Mobile Network as well as the Intelligent home Network with handover between them. The Virtual Network Functions on the NFV Layer identify the destination of IP packets and the SDN Controller directs these IP packets to their appropriate destination. 
Therefore our proposed solution will enable the building owner to have connectivity to different operators to facilitate the use of different devices registered with different operators, as well as exploiting the license-free spectrum for accessing the Internet or Mobile Network using the home network.

\section{A. RAN Architecture}

The Access Layer architecture uses a 10G Common Public Radio Interface (eCPRI) ring Ethernet, to interconnect a Upper Layer 1 processing on an FPGA with up to six Remote Radio Light Head (RRLH) Controller FPGAs each hosting two Lower Layer 1 processors, the first that generates an IF signal to drive up to 8 VLC MISO modules using a 1 to $8 \mathrm{RF}$ splitter/switch and a second that generates an IF signal to drive or be driven by up to $8 \mathrm{mmW}$ ave RF Duplex modules using a 1 to 8 RF splitter, as shown in Figure 3.

The functional split between the RRLH Remote Unit and the Central Unit in the Physical Layer 1 is at split 7 on the protocol stack [11]. The eCPRI Ethernet ring is looped from room to room in a building from one RRLH Controller to another in a similar way to the electric light circuit in a home.

A $10 \mathrm{MHz}$ GPS reference clock signal is sent to IHIPG, Upper Layer 1 Protocol Processor and RRLH Controller for use in $5 \mathrm{G}$ synchronization algorithms at these layers, thereby leaving just amplification and IF to RF up conversion to be performed at the RRLH.

As there is a limited amount of space available in Light Rose housing within which the VLC and mmWave RRLH is housed, the concept of Network Function Virtualisation (NFV) is adopted to off-load the complexity of the upper layer protocol processing of the communication systems required in the RRLH onto the IHIPG or CHDCS. This complexity consists of Network Layer 3, MAC Layer 2 and Physical Layer 1 processing.

DC-OFDM modulation is used for VLC transmission, which is compatible with the New Radio $5 \mathrm{G}$ frame formats.
The bandwidth of common VLC LEDs is up to $10 \mathrm{MHz}$ but this can potentially be extended to $100 \mathrm{MHz}$ depending on the quality of the LEDs lights used, which means that subcarrier spacing (SCS) of 15,30 and $60 \mathrm{kHz}$ from the $5 \mathrm{G}$ NR Frequency Range 1 (FR1) frame formats can be used, as shown in Table 5.3.2-1: Maximum transmission bandwidth configuration $\mathrm{N}_{\mathrm{RB}}$ for $\mathrm{FR} 1$ in [12], potentially providing maximum downlink bitrate of $691.2 \mathrm{M}$ bits/sec when 256QAM and $100 \mathrm{MHz}$ bandwidth is used. Since the IoRL project intends to use VLC LEDs of $10 \mathrm{MHz}$ bandwidth and SCS of $60 \mathrm{kHz}$, then this will provide a maximum downlink bitrate of $56.32 \mathrm{Mbit} / \mathrm{sec}$. The NR FR2 frame format, as shown in Table 5.3.2-2: Maximum transmission bandwidth configuration NRB for FR2 [12], can use much higher bandwidths of up to $400 \mathrm{MHz}$, using SCS of 60 or $120 \mathrm{kHz}$ and operating in 60 $\mathrm{GHz}$ unlicensed spectrum thereby providing uplink and downlink bit rates ranging up to $2.7 \mathrm{G}$ bits/sec, when using 256-QAM and $100 \mathrm{MHz}$ bandwidth, depending on the TDD frame type used. Since the IoRL project intends to use mmWave bandwidth of $100 \mathrm{MHz}$ and SCS of $60 \mathrm{kHz}$, then this will provide a maximum downlink or uplink bitrate of 675.84 Mbit/sec. The RRLH LED can be fitted with a range extension based on Plastic Optical Fiber, as shown on the lower VLC LED in Figure 3.

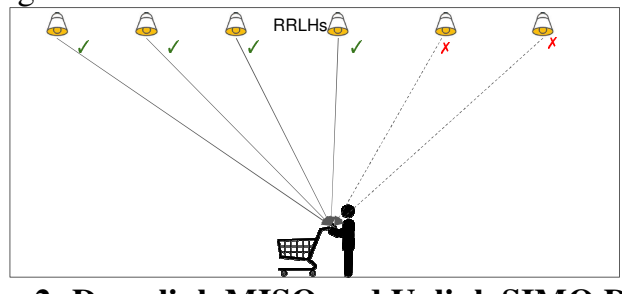

Figure 2: Downlink MISO and Uplink SIMO Diversity

MISO diversity is used in the downlink where the same data to be transmitted from different mmWave antennas / VLC LEDs by the RRLHs at the same time thereby increasing reliability. In effect this creates a manmade multipath

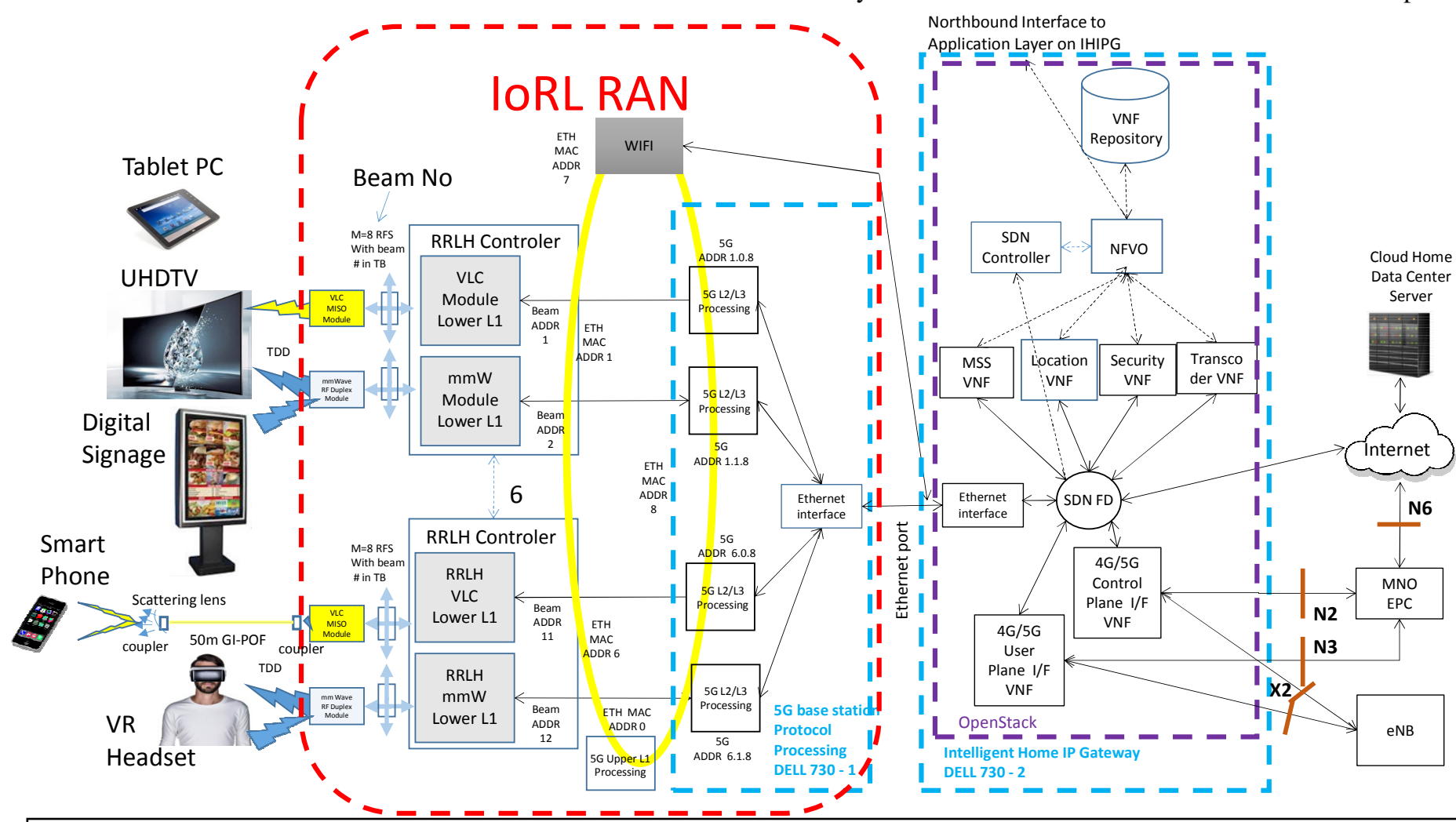

Figure 3: RRLH Architecture with External WLAN 
environment where if one or more of the light or mmWave paths is occluded then there is always the availability of the other paths to ensure continued communications, as shown in Figure 2. SIMO diversity will be used in the uplink where the same data is received by different mmWave antennas at the RRLHs and maximum ratio combined higher up in the layered protocol thereby increasing reliability.

In the case when all the paths are occluded, for example when someone conceals the Photo Diode (PD) receiver and mmWave antenna at the UE, then Multi Source (MS) streaming [13] is used to ensure that there is always the availability of another low capacity WLAN path for continued communications and continued synchronization with the streaming audio/video.

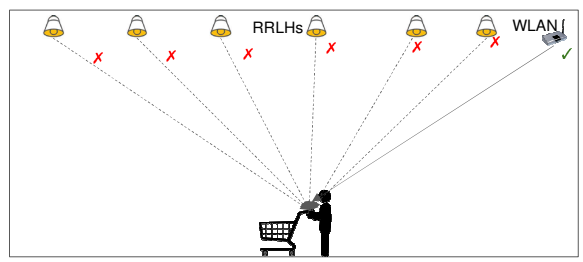

Figure 4: Multi Source Streaming to ensure connectivity

The Deep Packet Inspection NFV function is used at Intelligent HIPG to identify video streams and video transcoding is used to generate a lower quality Multi Source Stream (MSS) for the WLAN path to the UE, whereas the original higher quality SHDTV stream is transmitted by the broadband radio-light network [13]. This concept can also be extended to multipath TCP protocols.

\section{B. Software Defined Network Architecture}

The NFV Orchestrator (NFVO) is the top-level management entity of the IHIPG domain. The NFVO is the orchestration entity, which is responsible for the management of the Network Service (NS) lifecycle, which includes NS instantiation, dimensioning and termination. The NFVO receives appropriate commands from the upper layer (i.e. application layer) by use-case specific applications, which include the logic of each use-case and provides to the NFVO appropriate NS descriptors, which initiates the VNF instantiation with the appropriate network configuration internally in the IHIPG, which acts as a NFV Infrastructure (NFVI) Point of Presence (PoP).

A significant role of the SDN is to route IP packets within Ethernet MAC frames to/from the 5G L3 \& L2 Processing cores in the IHIPG depending on which RRLH Controller coverage area the UE is located and to/from Internet Bridge, 4G/5G Control Plane Interface and 4G/5G User Plane Interface depending on whether the IP Packets are Internet packets, 4G/5G Control packets or 4G/5G User packets, respectively. In the special case of video streaming, the SDN forwarding device is used to simultaneously route both higher and lower quality video streams to RRLH Controllers and WLAN. At the UE each of these streams is aggregated with each other to produce a video image of increasingly better quality as more and more streams are combined. Virtual Network Functions are used for transcoding the original high resolution video into lower resolution for transmission over the WLAN.
VLC and mmWave location estimation algorithms at the UE continuously report their location to the nearest $10 \mathrm{~cm}$ to a Location Service linked to the SDN Controller. Intra building handover between rooms or floors of a home network could either be performed by the MS Streaming application at the Service Level since its content consumption scheduler handles stream synchronization from multiple paths and multiple sources transmitted from different parts of the property or it could be performed at SDN Level by performing a handover between RRLHs depending on the measured location of the UE, as shown in Figure 5.

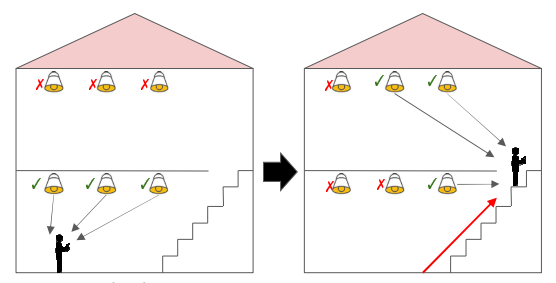

Figure 5: Intra Building Handover

The Reference Signal Received Power (RSRP) and Reference Signal Received Quality (RSRQ) of the outside radio network together with knowledge of the UE's location in the home radio-light network could be measured by the UE and reported to the LTE's Mobility Management Entity (MME) to initiate a conventional inter (between outside and building) network handover procedure.

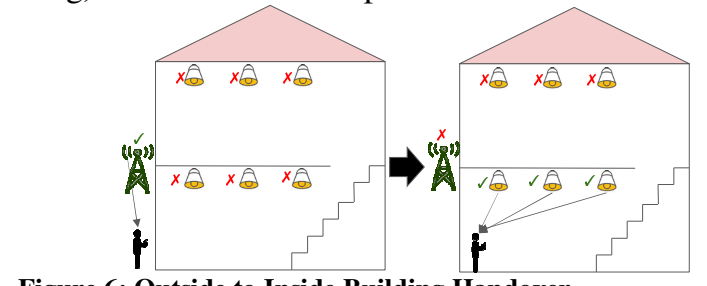

Figure 6: Outside to Inside Building Handover

\section{User Equipment Architecture}

The user equipment (UE) design is similar to the RAN design described in Section A above but clearly with much less computer processing power as shown in Figure 7.

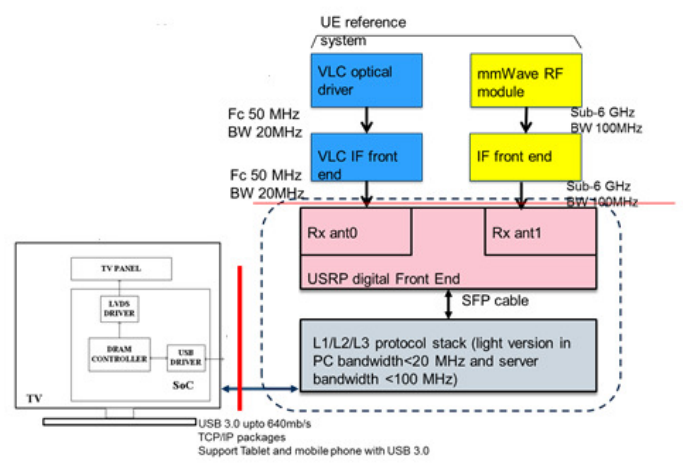

Figure 7: General scenario for VLC IPS

Using one RF chain of the RAN, a single VLC and mmWave antenna pair UE can be built with similar structure of the RAN. The mmWave card is used to convert between mmWave and radio frequency. The NI RF card can switch the RF signal to baseband I/Q signal and transmit it into the L1/L2/L3 protocol stack processing server for signal processing. Several UEs can be located at different positions in a room for multiuser access. 


\section{VLC based indoor positioning system}

In IoRL project, the positioning system consists of two parts: VLC-based positioning system and mmWaves-based positioning system. A high positioning accuracy, which is less than $10 \mathrm{~cm}$, could be provided by combining both techniques.

The positioning system based on VLC uses visible light signals for determining the positioning of target where the signals are transmitted by RRLH lamps (e.g. LEDs) and received by light sensors (e.g. photodiode (PD) or camera), on the target UE.

Eight reference amplitude sub-carriers on OFDM symbols from the Transport Block (TB) are dedicated to be uniquely sent by each of the eight lights in a MISO group. The received signal strength (RSS) at the UE PDs is proportional to the distance travelled from each of the light and can be used to estimate position from at least three distance measurements.

The positioning system based on mmWaves uses electromagnetic waves to determine the location of UEs. The location relevant parameters can be estimated either at UE (in the downlink) or at the RRLH controller (in the uplink).

In the uplink case, the UE is a transmitter. Multiple lamps (RRLHs) located at a priori known positions receive a signal transmitted from the UE. The RRLH controller performs measurements and estimates location relevant signal parameters such as the received signal strength (RSS), roundtrip times (RTTs), or the time-difference of arrival (TDOA) between different RRLHs. The time offsets caused by different cable length between mmWave antennas of each RRLH and the RRL controller must be a priori known to allow proper estimation of TDOAs and RTTs. In order to estimate RTT, the RRLH controller must transmit a signal over a particular RRLH and the UE must respond to it by transmission of localization signal. The response time of the UE must be a priori known at the RRLH controller for the proper estimation of the RTT in the uplink. The RTT estimation has to be performed successively for each RRLH.

In the downlink case, the UE is a receiver. Multiple lamps (RRLHs) located at a priori known positions transmit signals towards the UE. The UE performs measurements and estimates location relevant signal parameters for each of transmitted signals from RRLHs. The controller must generate orthogonal (time, frequency and/or code) signals that will be simultaneously received by the UE. The UE needs to estimate TOAs for each RRLH signal and compute TDOAs. As well as in the uplink, the time offsets caused by different cable length between mmWave antennas of each RRLH and the RRL controller must be a priori known to allow proper estimation of TDOAs and RTTs. In order to estimate RTT in the downlink, the UE must transmit a signal which is received by multiple RRLHs connected to the RRLH controller. The RRLH controller responds to UE using orthogonal signals over multiple RRLHs. The response time of the RRLH must be a priori known at the RRLH controller for each RRLH which is connected to it. The UE is estimating TOAs for each RRLH signal and computes corresponding RTTs.

In both, the uplink and the downlink case, the estimated parameters are communicated to the CHDCS (Cloud home data control server) which location related service calculates locations of UEs.

\section{CONCLUSIONS}

A 5G Radio-Light system has been described that is so flexible that it can be easily expanded if required to support home and business properties that vary in size from small to large and that can provide sufficiently large bandwidth to support high quality digital media for numerous people in a building using broadband services. The architecture is sufficiently flexible to support different types of applications in homes and businesses. It operates in unlicensed spectrum so that there are no restrictions to deploying it (i.e. does not require the permission of MNOs). The architecture that is so simple and similar to the electric light circuit in properties that it is expected that the ordinary electrician can install it in order to easily install it in a large number of properties. An appealing set of example use cases are presented of the type of broadband services that can be provided in homes using IoRL technology.

\section{Acknowledgments}

The authors gratefully acknowledge the financial support of the EU Horizon 2020 program towards the Internet of Radio-Light project H2020-ICT 761992.

\section{References}

[1] Rima Qureshi “Ericsson Mobility Report” June 2016.

[2] Office of National Statistics (ONS) http://www.ons.gov.uk/ons/taxonomy/index.html?nscl=B usinesses+by+Size\#tab-data-tables.

[3] "The Cisco Visual Networking Index (VNI) Global Mobile Data Traffic Forecast Update", Cisco, Feb 2016

[4] "Amdocs 2015 State of the RAN", Amdocs, 2015

[5] "Wireless in Buildings", Commscope, February 2016

[6] "In-Building Wireless", ABI Research, April 2014 / "Wireless in Buildings", Commscope, February 2016

[7] T. S. Rappaport et al., "Millimeter wave mobile communications for $5 \mathrm{G}$ cellular: It will work!" IEEE Access, vol. 1, no. 1, pp. 335-349, Aug. 2013.

[8] Sridhar. Rajagopal, Shadi. Abu-Surra, Sudhir. Ramakrishna, et al "MIMO designs for mmWave wireless LAN systems" 48th IEEE Signal Proc Soc. Asilomar Conference on Signals, Systems, and Computers, Pacific Grove, CA Date: Nov 02-05, 2014, Pp: 1916-1920

[9] H. Zhang, X. Chu, W. Guo, S. Wang "Coexistence of Wi$\mathrm{Fi}$ and Heterogeneous Small Cell Networks Sharing Unlicensed Spectrum" IEEE Communications Magazine, Vol. 53, Iss. 3, Pp:158-164, Mar 2015.

[10] John Cosmas, Yue Zhang, Xun Zhang, "Internet of RadioLight: 5G Broadband in Buildings," European Wireless 2017, Dresden, Germany, $17^{\text {th }}-19^{\text {th }}$ May 2017.

[11] S. Perrin "Evolving to an Open C-RAN Architecture for 5G" Fujitsu Heavy reading White Paper, September 2017

[12] 3rd Generation Partnership Project; Technical Specification Group Radio Access Network;NR;Base Station (BS) radio transmission and reception(Release 15) - 3GPP TS 38.104 V1.0.0 (2017-12)

[13] J. Bruneau-Queyreix, M. Lacaud, D. Negru, J. Batalla, and E. Borcoci, "MS-Stream: A Multiple-Source Adaptive Streaming Solution Enhancing Consumers Perceived Quality," in IEEE Consumer Communications and Networking Conference (CCNC) - Best Demo award, 2017. 